\title{
An Opportunistic Dissemination Protocol for VANETs
}

\author{
Amina SEDJELMACI \\ Dept. of Telecommunications \\ University of Tlemcen, Algeria
}

\author{
Fedoua DIDI \\ Dept. of Computer Engineering \\ University of Tlemcen, Algeria
}

\author{
Ahmed ABDUL RAHUMAN \\ Dept. of Electronic Engineering \\ University of Tlemcen, Algeria
}

\begin{abstract}
In this article, we propose an Opportunistic information dissemination protocol by mixing both flooding and an enhanced DHVN (Dissemination protocol for heterogeneous Cooperative Vehicular Network) protocol, allowing them to run opportunistically in a Manhattan plan. Special additional logic is added to the existing version of DHVN protocol in order to efficiently disseminate information in two steps: 1) by adding three tags, Initial Diffusion, Standard DHVN and DHVN Near Intersection; the Initial Diffusion tag is used for the first flooding transmission only and 2) by changing the SNF (Store and Forward) period by making it adaptive depending on the region. Detailed simulation results show that our opportunistic protocol outperforms the DHVN protocol by analyzing its performances using an integrated framework VNS.
\end{abstract}

Keywords-Flooding; DHVN; SNF; opportunistic; VANET

\section{INTRODUCTION}

In recent years, the dissemination of data in VANET (Vehicular Ad hoc NETworks) has attracted a lot of attention given its imminent role in improving road safety and reducing traffic congestion. The issues weigh heavily on the economy of a country in terms of energy and time. To be able to reduce the risk of accidents, avoid dangerous situations and mitigate such problems, we have to succeed in effectively disseminating relevant information and spreading it as far as possible with a minimum bandwidth usage.

The way in which relevant information is broadcasted throughout the vehicle environment is considered as a most important aspect for the vehicles cooperation in VANETs. However, several problems can occur during this process of dissemination: (1) an excessive consumption of bandwidth in the case where we are confronted to an urban area. (2) A disconnected network problem can occur in the case of a rural area. These problems constitute a crucial challenge and the question that will arise to face them is "what can we do to overcome the rural disconnection without excessive use of bandwidth and how can we avoid broadcast storms while keeping a high coverage ratio?"

In this paper, we attempt to address these issues by suggesting an improved opportunistic DHVN protocol that we call oDHVN. The remainder of this paper is organized as follows. Section II describes related works about different dissemination protocols operation mode classes. Both simulation tool and scenarios are given in Section III. In Section IV, our opportunistic protocol is described. Simulation results of the designed solution are presented and discussed in Section V. Finally, Section VI concludes the paper.

\section{RELATED WORKS}

An interesting aspect of VANETs is that most of their applications require an efficient and reliable multi-hop data broadcast protocol, making this task performed by the protocol one of the most difficult and indispensable network functions. For example, avoidance of vehicle collisions and post-crash warnings require efficient and robust data dissemination, especially when the distances between the sender and the intended receivers are greater than the radio transmission range [1]. We focused on approaches that focus on reducing bandwidth usage by limiting the number of re-transmissions with optimal selection of relays and transmission parameters based on network conditions.

\section{A. Characteristics and Operation Mode Classes}

Data dissemination approaches in VANETs can be classified into three main classes: Relayed Instantaneous Broadcast, broadcast with store-and-forward SNF relay, and opportunistic broadcast. In the Relayed Instantaneous Broadcast approaches, the data is broadcasted to all neighboring vehicles that will briefly store the data and then a neighbor vehicle is selected as a relay to rebroadcast it in turn. This approach works well in high density networks that avoid broadcast storms, but require efficient relay selection to ensure reliability. A good survey on this class can be found in [2].

Alternatively, in the broadcast with store-and-forward SNF relay approach, data is stored, transported and then rebroadcast on network partitions, making them more suitable for irregularly distributed vehicle density zones. In the opportunistic approach, the two previous approaches are combined to adapt according to the circumstances of the network (dense, disconnected, high occupancy rate of the channel, etc.).

Other classifications that are based on other functional aspects can be found in the literature. In [1], flooding is considered a full-fledged approach whereas it can be defined as a special case of Relayed Instantaneous Broadcast approaches since the data is disseminated to all neighboring vehicles which are all considered as potential relays for a single retransmission (there is no store and forward) to their neighboring vehicles.

In [3], the first two approaches are grouped into one approach creating two classes; stateless broadcasting and stateful broadcasting. In the first class, there is no need to 
obtain information about the network topology while the second class, the protocol requires information on the local topology.

In [4], the authors distinguish two main categories: multihop broadcast and single-hop broadcast. The authors divide the first class into several subclasses according to the method of election of the relay (delay, probability) or according to the use of the method "network coding" [5]. In the single-hop broadcast, when receiving a message, the vehicle retains and updates the information in its embedded database and in turn broadcasts, periodically in its one-hop vicinity, its version of the information. Authors divide this class into two subclasses depending on whether the diffusion decision period is fixe or adaptive.

In a more recent work [6], the authors repeat the general multi and single hop classification but adopt another reasoning more in line with ours. In this last work, the focus is on the multi-hop class which includes the largest number of protocols where the authors consider two different categories: 1) restrictive methods, and 2) promiscuous methods. As for our classification's first class, restrictive methods combine techniques to address the problems of broadcast storms. The difference from previous work lies in the introduction of the promiscuous class subclass where VANETs can be fragmented and partitioned, hence the use of techniques such as Store-andForward to ensure that the information is correctly disseminated. The authors mention other approaches (not clearly classified) that combine two different techniques to improve dissemination performance. These correspond to our third class of opportunistic diffusion. Other classifications based on different points of view or spanning other higher spheres (Security, QoS, Encryption, Topology, etc.) can be found in [7], [8].

\section{1) Relayed Instantaneous Broadcast class}

One of the representative protocols of this class is the Distance Defer Transfer Protocol (DDT) [9]. In DTT, upon receipt of a new message, the vehicle triggers a timer that is inversely proportional to the distance to the transmitter. During this waiting time, the vehicle records the positions of all vehicles that transmit the same message and decides to abandon the retransmission if most of its own retransmission area has been sufficiently covered by its neighbors. Otherwise the vehicle retransmits the message by applying the same protocol.

Another representative protocol of the first class can be found in [10] where the original transmitter simply accesses the medium using the standard 802.11 CSMA / CA technique and broadcasts the entire emergency message. All neighboring vehicles in the transmission range calculate their corresponding SNR values as well as their Euclidean distance from the source via GPS. Subsequently, each receiver then uses these results to calculate the maximum size of the specific contention window (CWmax). Each node randomly chooses a time slot in the range $[0, \mathrm{CWmax}]$ and waits during this slot time. The node that chooses the shortest time interval becomes the relay and rebroadcast the emergency message. The rebroadcast message serves as an acknowledgment to the original sender.
In [11], the SIFT protocol is proposed and comprises two phases: 1) the trajectory calculation which is only executed by the source node before sending a new packet for the first time. This phase calculates the trajectories and sends the packets by triggering the multi-hop routing process. 2) The packet routing phase that is invoked by each intermediate node when receiving a packet. This phase allows the node to decide by triggering a timer according to its position with respect to the trajectory and the transmitter whether or not to transmit the packet.

In [12], the selection of the next relay is performed by the calculation of a probability by each receiver of an emergency message. The latter will determine a Backoff period (i.e. the waiting time before retransmitting the received message). The backoff duration is calculated according to the following formula: Where WT is the value of Backoff, CW is the contention window, $\mathrm{P}$ is the calculated probability.

$$
W T=C W \times(1-P)+\delta
$$

In this way, the vehicle with the shortest waiting time will transmit the message first. The vehicle with the highest probability will have the shortest backoff period. This retransmission probability is a weighted sum of two parameters: the distance factor (D) and the link quality factor (LQ). It is calculated as follows:

$$
P=\left(1-\omega_{P}\right) D+\omega_{P} L Q
$$

Where $\omega_{P}$ is a weight between 0.5 and 1 . This is to give more importance to the quality of the link, since the security messages considered in this study are critical in nature and the reliability of the transmission is one of our main axes.

In [2], The authors take a new approach to the calculation of the waiting time and use ZigBee as a communication technology to eliminate the redundancy of broadcast messages and thus minimize the Storm Broadcast. Indeed, the waiting time is adjusted according to the distance of the vehicle from the base vehicle and the relative speed. If the vehicle speed is slow, its waiting time is increased. The vehicle that travels at the highest speed and has the largest number of nearby vehicles will have very little waiting time and will be broadcast instantly.

\section{2) Broadcast with store-and-forward SNF relay}

There are several protocols in the literature that belong to the second class, broadcast with SNF relay type. In (Cherif et al., 2010) [13], the ROD protocol is organized in two modules; 1) an ODDT module (Optimized Distance Defer Transfer): the same method as in the DDT is adopted where the GPS position of the vehicle is encoded in the header of the broadcast message. The ROD protocol encodes additional information in addition to the GPS_pos which represents the position of the sender, OI_pos represents the outbound intersection position and II_pos represents the incoming intersection position that will be used by the ODDT module to optimize the data dissemination, in sections of road (between two intersections) and in intersections. 2) The Store and Forward SNF module where if no relay vehicle is found due to temporary network 
fragmentation, the vehicle in charge of the message uses the Store and Forward module to keep the data until a better retransmitter is found.

Another protocol representative of the broadcast class with store and forward relay is the DHVN protocol [3] which gives particular attention to the network connectivity, the road structure and the heterogeneity of the vehicles. In this protocol, the source car broadcasts the packet in both directions where each receiver on the same route triggers a timer based on the distance to the transmitter. It retrieves the sender's position information from the packet header and calculates the backoff delay as follows:

$$
T=\frac{1}{\text { dist }+ \text { car_height } * M D}
$$

Where dist is the distance between sender and receiver, Car_height is the height of the vehicle, $M D$ is the maximum extra distance when the vehicle is 1 meter higher than a standard vehicle. A relay is chosen for each route and each direction to propagate the message. Since vehicle networks are also highly partitioned networks, continuous connectivity is not guaranteed. To allow longer-term dissemination of information in the case of highly partitioned networks, the DHVN protocol uses the SNF approach where nodes carry information with their movement and transmit it periodically. In DHVN, the choice of the retransmission period is crucial. Indeed, a small period causes a loss of bandwidth and a high period implies a significant delay. The algorithm of DHVN protocol is summarized in Fig. 1.

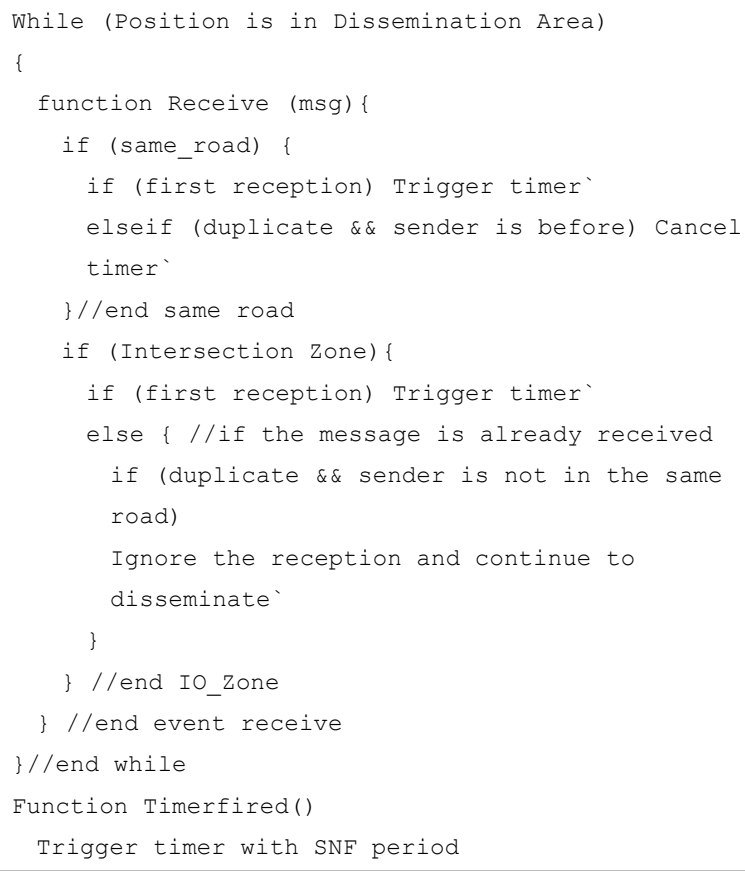

Fig. 1. Original DHVN protocol algorithm.

The TrAD protocol [14] requires beaconing to maintain a list of vehicles and their status in a single-hop neighborhood in order to work seamlessly in both urban and rural scenarios. It is composed of two main components: 1) The flooding suppression technique for a well-connected network which makes it possible to constrain the broadcast storm problem and improve the reliability of the transmission; and 2) the SCF mechanism (Store-Carry-Forward) that selects the appropriate vehicles to act as relay which save the message and rebroadcast it in a disconnected network. Several concepts of TrAD are defined as (a) Directional cluster: It is a group of vehicles in the neighborhood of a sender $\mathrm{S}$, which are in a similar direction with respect to the sender S. (b) Coordinator: the coordinator is the vehicle that is located at an intersection. (c) Breaker: In a well-connected network, the breaker is not only the farthest vehicle but also the one that moves out of the network.

The first component contains two mechanisms: 1) Classification of vector-angle clusters which consists of designing several clusters according to the vector angle with the sender $\mathrm{S}$ to identify if the vehicles belong to a directional Cluster. 2) Traffic-adaptive sorting technique that takes account both for road traffic and network traffic status and for assigning the transmission task to the neighbor who has both a dense neighborhood, a greater distance from the sender and the lowest rate of transmission (occupation of the canal).

The second component also includes two mechanisms: 1) Selection of the SCF agent: this technique makes it possible to identify the breaker; when the vehicle receives a data message, the protocol checks and eliminates the possibility of being a coordinator. After that, the vehicle checks whether its direction of travel is the same as the direction of the data. If so, the vehicle will look for another neighbor even further, which also moves in the direction of data transmission. Otherwise, the vehicle is defined as a breaker. This procedure will go to the limit of the connected network. 2) SCF redundant redistribution technique: This technique is intended to trigger the re-broadcast of the SCF or restrict it if more than one SCF receives the same request. Thus, a different broadcast delay for each SCF is calculated based on the shortest distance of the sender and the lowest channel occupancy.

\section{3) Opportunistic broadcast.}

Rare are the protocols that belong to this class [15], [1]. Such protocols take advantage of the strengths of the two first classes and mitigate their weak points by combining them to propagate data via a vehicular ad hoc network (VANET). The authors in [15] propose a hybrid protocol that allows to merge the two classes by classifying the field relative to each vehicle as a so-called multi-hop broadcast MHB area or a so-called store and forward (SF) area. The MHB and SF regions are partitioned via a broadcast area radius $(\mathrm{R})$ around the sender where the data is broadcasted via a multi-hop broadcast in the broadcast area. Outside the MHB area, the message spreads via the store and forward protocol.

\section{Methodology}

\section{A. Simulation Tool}

Two aspects of the VANET simulation exist, the first aspect concerns the simulation of the vehicles mobility and the second concerns the simulation of the network components. Mobility simulators have the ability to generate realistic vehicular mobility traces reflecting the movements of vehicles. These will be introduced as input for the network simulator. 
There are several mobility simulation software environments and their main characteristics are: a) Supported trace formats, b) Roadmap type, c) Supported mobility model, d) Implemented traffic model.

Network simulators can be used to simulate network components in a detailed way such as source, destination, channel and data traffic transmission. The main features of the network simulator are a) large-scale simulation capability, b) ease of installation and use, c) MAC protocols and supported networks. For the network components simulation, we use NS3 which is a discrete-event network simulator for Internet systems targeted primarily for research and educational use [16]. Nowadays, NS-3 is still evolving where some models are still under development. However, the NS-3 simulator remains one of the smartest choices like OMNeT ++, JISt are. We chose NS-3 because it is a free and open source software, for its potential to expansion that materializes in the large and growing number of source code contributed by the NS-3 computing community and for its extendibility and stability. To allow easy integration of new models in NS-3, its network architecture is inspired by the real world in terms of hardware and software.

Finally, the VANET or Framework simulators allow integration and coupling between the network simulator and the traffic simulator. The degree of integration level will or will not allow the vehicle to change direction or leave the road in response to a network event.

Some popular platforms of vehicular networks simulation can be cited such as VEINS [17], [18], iTETRIS [19]-[21] and VNS [22] which are considered as very powerful platforms to simulate and evaluate VANETs protocols.

The interface module of the first two platforms which is used to interconnect both traffic and network simulators, introduces a communication and synchronization overhead, consequently reducing the efficiency of the global simulator. On the other hand, VNS proposes a unique framework which makes it possible to avoid additional calculation overhead by completely integrating the aforementioned NS-3 network simulator and the traffic simulator DIVERT 2.0.

Various realistic roadmap formats can be imported by DIVERT 2.0. It also provides vehicle models with different driver behaviors as well as a realistic traffic generation model.

\section{B. Simulation Scenario}

In VNS, the author proposes a modified version of the Manhattan model where traffic lights are present at each intersection. Several lanes are present at each street. The Divert 2.0 mobility model defined in VNS introduces elements relating to the characteristics of a micro-mobility model such as the driver model in each vehicles that offers more realism. The rate of creation of the vehicles is given by the user.

This modified version of Manhattan in VNS is a result of a manifestation of an initial transitional period that depends on the size of the map (number of streets). This period is followed by a stationary vehicle density and in which the simulation results are taken into account.
Our simulation was structured as follows:

- The transformation into parameterized variables of several simulation parameters considered important and which are likely to change during the simulation.

- The ability to change the simulation parameters from the command line.

- The choice to be able to control the simulation by means of a python script.

- The evaluation and the tracing of the results are done by the use of the library Scipython.

We performed a VNS simulation using the input parameters represented in Table I. The first dissemination message is triggered randomly after the fulfillment of the following condition:

$$
n b r_{-} V \geq T r u n c\left(\frac{n b r_{-} L * l e n_{-} L * d e n s_{-} V}{100}\right)
$$

To avoid the initial transition period of the simulation, we established this formula empirically after several attempts. To avoid re-executing the simulation several times, the sending of a new emergency message is retriggered each time period defined by the "time_to_send_new_message" parameter. For each transmission, the triggering vehicle must be within 30 meters of the center of the map.

TABLE I. INPUT PARAMETERS OF A VNS SIMULATION

\begin{tabular}{|c|c|}
\hline Input parameters & Definition \\
\hline gui & $\begin{array}{l}\text { controls the visualization of the result as } \\
\text { an animated graph }\end{array}$ \\
\hline finishtime & control the duration of the simulation \\
\hline Nbr_L & sets the roads number \\
\hline len_L & $\begin{array}{l}\text { defines the length of the slices of roads } \\
\text { and thus the size of the blocks (block of } \\
\text { house) }\end{array}$ \\
\hline dens_V & $\begin{array}{l}\text { defines the rate of creation of vehicles on } \\
\text { each road }\end{array}$ \\
\hline time_to_send_new_message & $\begin{array}{l}\text { sets the minimum time between two } \\
\text { sendings of a new emergency message }\end{array}$ \\
\hline maxspeed & Sets the maximum speed for each route \\
\hline normal_range & $\begin{array}{l}\text { defines the standard height vehicle } \\
\text { reception range }\end{array}$ \\
\hline high_range & $\begin{array}{l}\text { sets the highest vehicle receipt range } \\
\text { (Bus, Truck) }\end{array}$ \\
\hline high_vehicle_ratio & the percentage of high vehicle creation \\
\hline dhvn_snf & $\begin{array}{l}\text { sets the period of transmission of the } \\
\text { message by the relay DHVN }\end{array}$ \\
\hline dhvn_message_TTL & The lifetime of the message \\
\hline
\end{tabular}

\section{Evaluation Parameters}

We performed simulations for the DHVN protocol in an urban and rural environments. The different simulation parameters are represented in Table II. 
TABLE II. SIMULATION PARAMETERS OF DHVN PROTOCOL (URBAN AND RURAL)

\begin{tabular}{|l|l|}
\hline Parameters & Values \\
\hline Simulation zone & $\begin{array}{l}1200 \mathrm{~m} * 1200 \mathrm{~m} \text { in urban and } \\
3000 \mathrm{~m} * 3000 \mathrm{~m} \text { in rural }\end{array}$ \\
\hline Number of Road & 5 \\
\hline Length of the Roads & $\begin{array}{l}500 \mathrm{~m} \text { in rural and } 200 \mathrm{~m} \text { in urban }(5 \times 5 \\
\text { roads) }\end{array}$ \\
\hline Number of nodes & $\begin{array}{l}700 \text { a } 1750 \text { in urban and } 2000 \text { à } 3000 \\
\text { in rural }\end{array}$ \\
\hline The propagation model & $\begin{array}{l}\text { Three Log Distance Propagation Loss } \\
\text { Model and Nakagami propagation loss } \\
\text { model with two variations of emission } \\
\text { power for each type of vehicle height }\end{array}$ \\
\hline Vehicle height & $\begin{array}{l}1 \mathrm{~m} \text { (ordinary vehicle) -- 2m (truck or } \\
\text { bus) }\end{array}$ \\
\hline High vehicle rate & 20\% \\
\hline Coverage area (Radio Range) & $\begin{array}{l}250 \mathrm{~m} \text { (ordinary vehicle) --375 m } \\
\text { (truck or bus) }\end{array}$ \\
\hline
\end{tabular}

To evaluate the performance of the DHVN protocol, we will focus on the following metrics:

- Coverage which equals the total number of successful first receptions divided by the estimated number of vehicles in circulation.

- The average number of duplicate reception which is equal to the total number of duplicates receptions for a message divided by the estimated number of vehicles in circulation.

- Performance index, which is calculated according to the formula $\frac{\text { coverage }^{2}}{\text { Duplicate_reception_average } / 3+100}$ where we have emphasized the importance of coverage and reduced that of duplicate reception. We will rely on this index, whose values vary between 0 and 100, for the choice of the optimal result.

\section{OUR PROPOSITION}

\section{A. Exploring Some Enhancement Approaches}

We performed a simulation of the DHVN protocol for different SNF periods and obtained the results of both urban and rural environments as illustrated in Fig. 2 and 3, respectively. We can clearly see that the performance of the DHVN protocol has great sensitivity to the SNF period with the performance index varying between 26 and 51 for the urban environment and between 20 and 54 for the rural environment. The Best results are obtained for an SNF period of $45 \mathrm{~s}$ and $20 \mathrm{~s}$ for the urban and rural environment respectively. This is apparently tied to the environment (lane length, intersection density and vehicle density) and the DHVN protocol user must choose the SNF period very carefully. In our modified algorithm, we will try to attenuate this sensitivity and find a logic to select an adaptive SNF period without the intervention of the user.
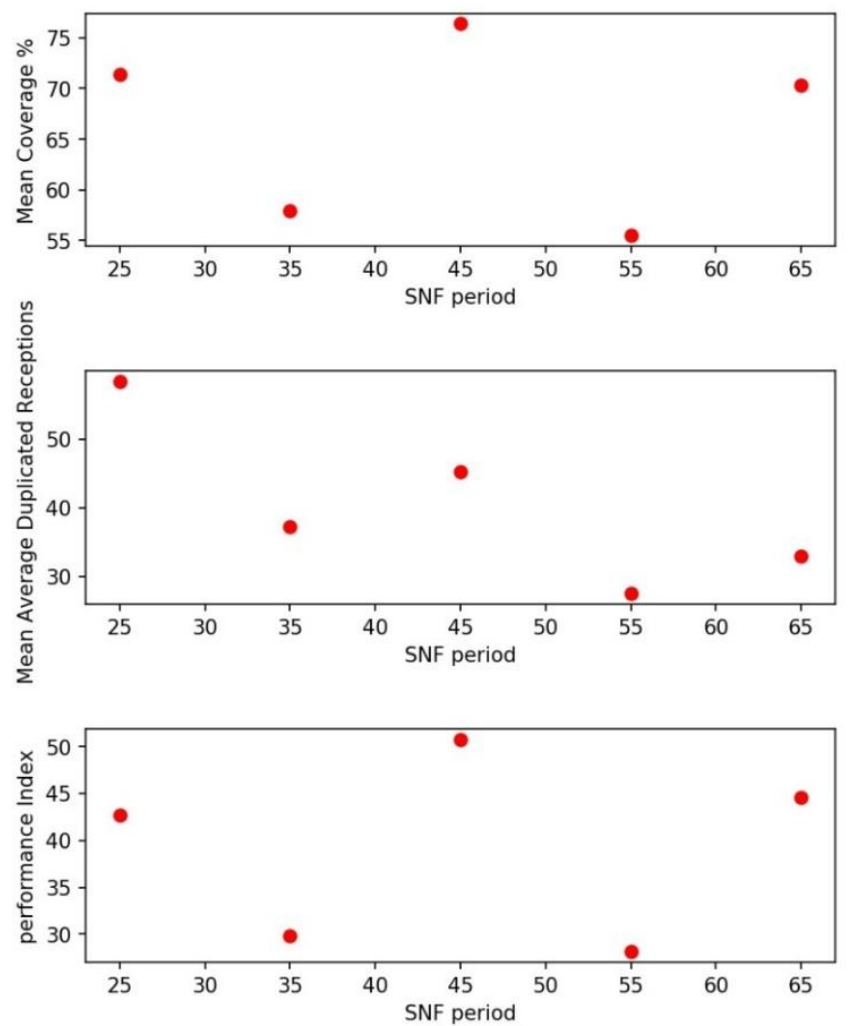

Fig. 2. DHVN protocol simulation results for different SNF periods in urban environment.
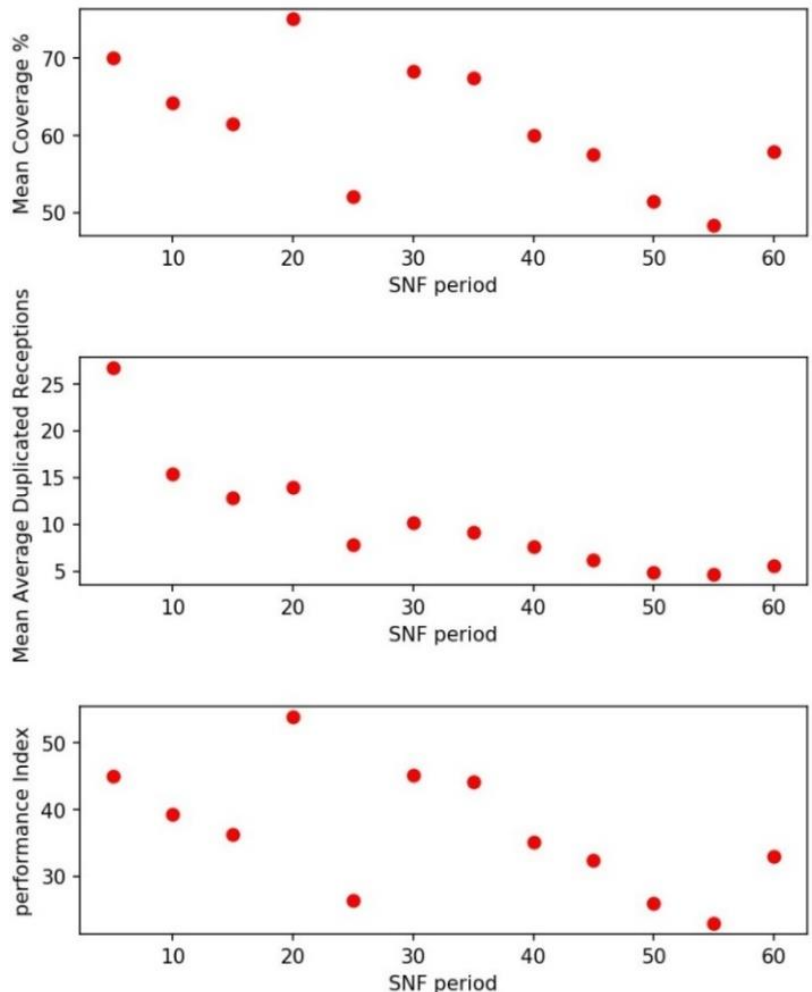

Fig. 3. DHVN protocol simulation results for different SNF periods in rural environment. 
Additionally, as we can see in Fig. 3, in some special cases where the original transmitter is in very low vehicle density zone, the DHVN protocol misfires and resulted performance is very poor. This is very likely the case for the SNF periods where the coverage is lower than $50 \%$.

Another cause for these poor results would be tied to SNF period termination being triggered in a very low vehicle density zone. This means that the relay vehicle would be in the waiting state while passing high density zones like road intersections. We also consider this case to be a DHVN protocol misfire. On the other hand, in urban environment where mostly all the zones are high vehicle density ones, the DHVN protocol produces too many duplicate messages which means a high wastage of the transmission bandwidth. We will be taking these observations into account in our enhanced algorithm by opportunistically synchronizing the transmission of the DHVN message with the passage of the relay near an intersection zone.

To further explore the DHVN protocol performance variation, we performed a simulation for different TTL durations and an SNF period equal to 45 seconds in the urban environment and equal to 20 seconds in the rural environment. The obtained results of both urban and rural environments are illustrated in Fig. 4 and 5, respectively.

We can observe again a certain sensitivity of the resulted performance to the TTL duration with the performance index varying between 36 and 54 for the urban environment and between 11 and 61 for the rural environment. The best results are obtained for the TTL duration of $150 \mathrm{~s}$ and 400s for the urban and rural environment, respectively. This is easily explained due to the SNF mechanism; a short TTL duration doesn't allow for the message to propagate far enough, while a large TTL duration results in excessive bandwidth usage. So logically, an ideal TTL duration would be a function of the size of the dissemination area and the mobility model (speed, acceleration, stop sign waiting time, etc.). We will not be introducing a selection mechanism in our enhanced algorithm but we will be using these values for further simulation so we can have the best performance results.

Another aspect of the original DHVN protocol that can be criticized is the use of the distance between the receiver and the transmitter to calculate the backoff delay. While in an ideal scenario, the DHVN relays would be moving away from the position where the first message originated; this is especially not true in a sparsely distributed road map. So we had the idea of calculating the backoff delay using the distance between the receiver and the position of the original transmitter. This later position is preserved in the transmitted DHVN message.

\section{B. Final Detailed Algorithm}

The resulted enhanced algorithm for the Opportunistic DHVN is illustrated in Fig. 6 with D_to_inter is the distance of the vehicle to the next intersection, T_Range is the transmission range of the vehicle and SNF_P is the adaptive SNF period.
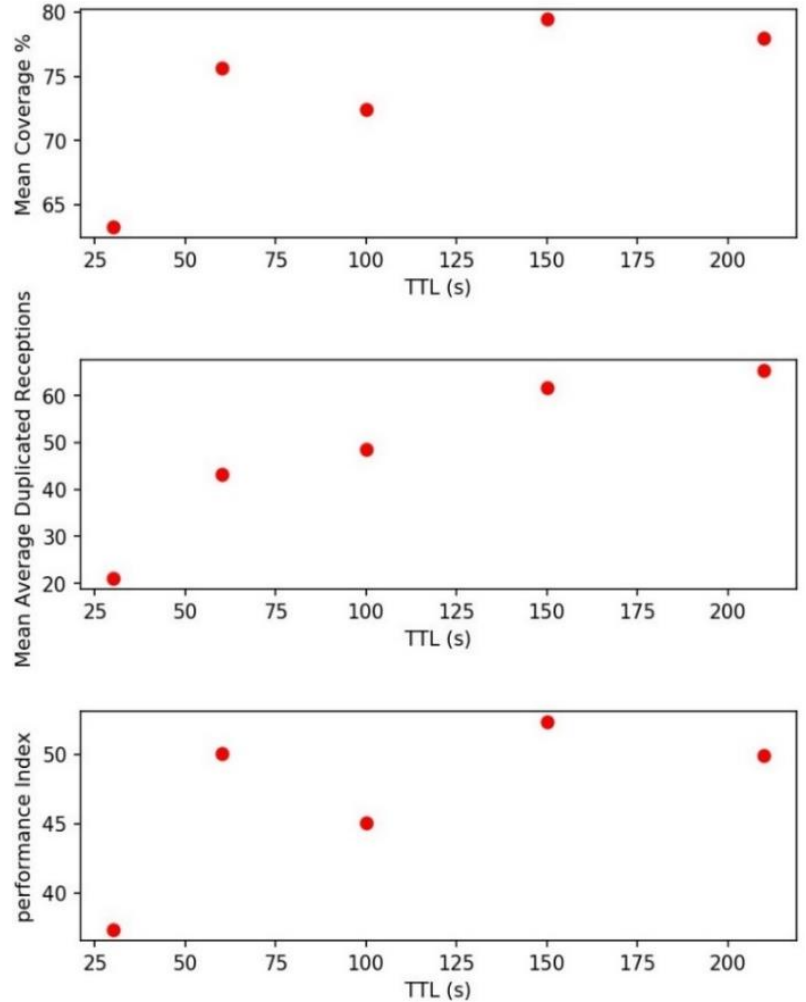

Fig. 4. DHVN protocol simulation results for different TTL duration and SNF periods of $45 \mathrm{~s}$ in urban environment.
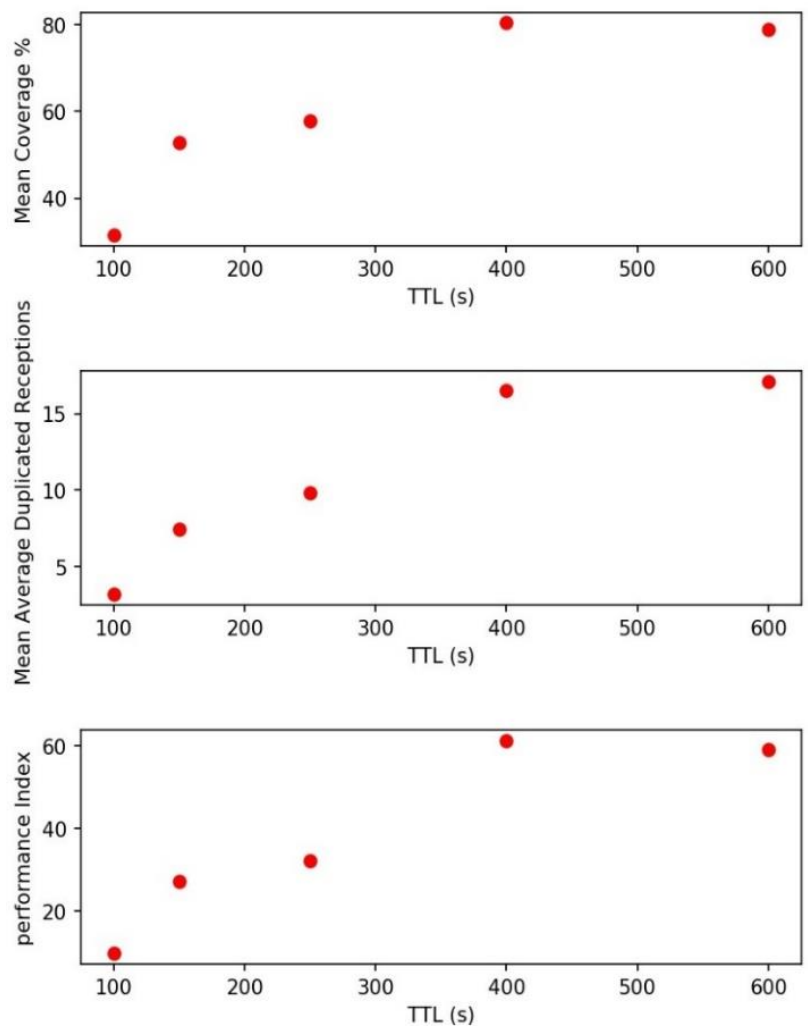

Fig. 5. DHVN protocol simulation results for different TTL duration and SNF periods of 20 s in rural environment. 


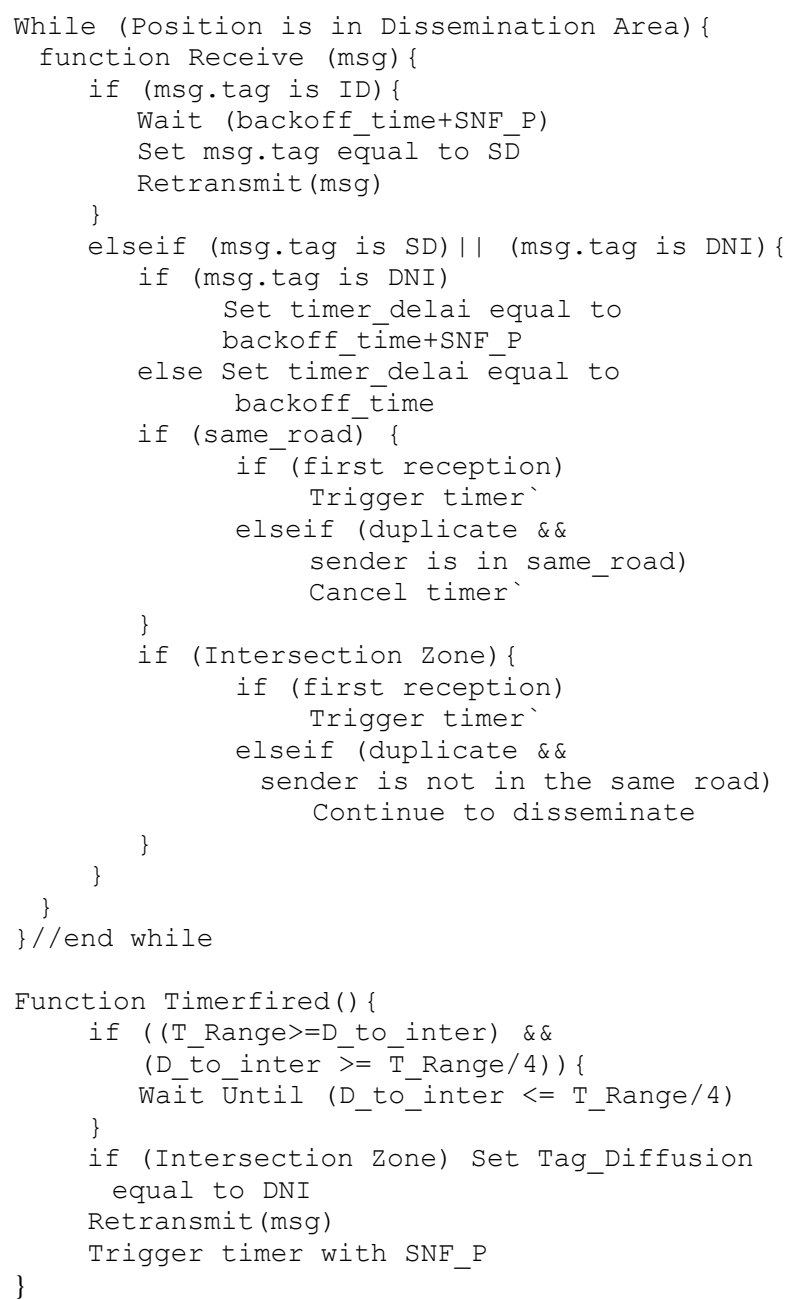

Fig. 6. Opporunistic DHVN protocol algorithm.

The algorithm introduces the following changes:

- Three tags are introduced: Initial Diffusion (ID), Stranded DHVN (SD) and DHVN Near Intersection (DNI). The Initial Diffusion tag is used for the first transmission only while DNI tag is used if the relay is inside an intersection. The SD tag is used elsewhere.

- The condition "Same road" cancels the DHVN Timer instead of "Same road before".

- An adaptive SNF period is introduces and is calculated as (Transm_Range/V_max_speed).

- If the vehicle relay is close to an intersection (T_Range $\leq$ D_to_inter $\geq T_{-}$Range/4) and the SNF period termination is triggered, the transmission is delayed until the relay is about to inter the intersection (D_to_inter< T_Range/4).
- In the first DHVN retransmission the vehicles wait for Backoff_Time+SNF_period instead of just Backoff_Time to counter balance the Initial Diffusion flood message.

\section{RESUlTS AND DISCUSSION}

We performed a simulation of the new oDHVN protocol for different TTL durations taken around the previously outlined 150s and 400s TTL durations for the urban and rural environments, respectively. The obtained results are illustrated in Fig. 7 and 8, respectively.

In the case of the urban environment and except for the very law TTL duration of 50s, we can observe a stable performance index around 60 with the best result being 65 for the TTL duration of 200 s.

In the case of the rural environment we can observe a slightly larger variation of the performance index between 56 and 77 with the best result being for the TTL duration of 600s.

We can clearly observe the enhancement in the performance index value and stability. Additionally we didn't have to choose an SNF period which gives the introduced oDHVN protocol a great advantage in autonomy and ease of use.

\section{CONCLUSION}

In this work we studied a palate of the VANET information dissemination protocols that we classified into three major classes, Relayed Instantaneous Broadcast, broadcast with storeand-forward SNF relay, and opportunistic broadcast. We underlined the performance of a recently introduced algorithm in [3], which is the DHVN protocol. We consider the latter to be a very good representative of the second class of dissemination protocols and we chose it as a basis for a new enhanced opportunistic dissemination protocol, the oDHVN.

Our contribution is represented by the enhanced algorithm which we have taken as the basis for our new oDHVN protocol. This algorithm was the fruit of well thought analysis of the behavior and performances of the standard DHVN protocol in both urban and rural environments. We have presented the results of the simulations carried out on the VNS framework for both the standard DHVN and the introduced oDHVN.

These results state clearly the enhancements brought by the oDHVN protocol. These enhancements are observed, on one hand, in the maximum value of the performance index which translates an equilibrium between the coverage percentage and the bandwidth usage. They are also observed, on the other hand, in the overall stability of the performance indicators. 

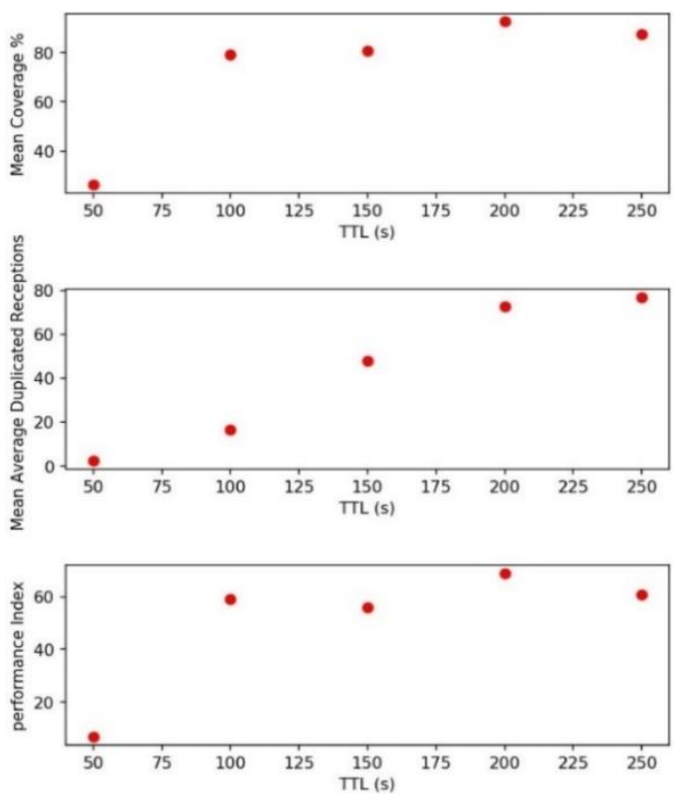

Fig. 7. Opportunistic DHVN protocol simulation results for different TTL duration in urban environment.
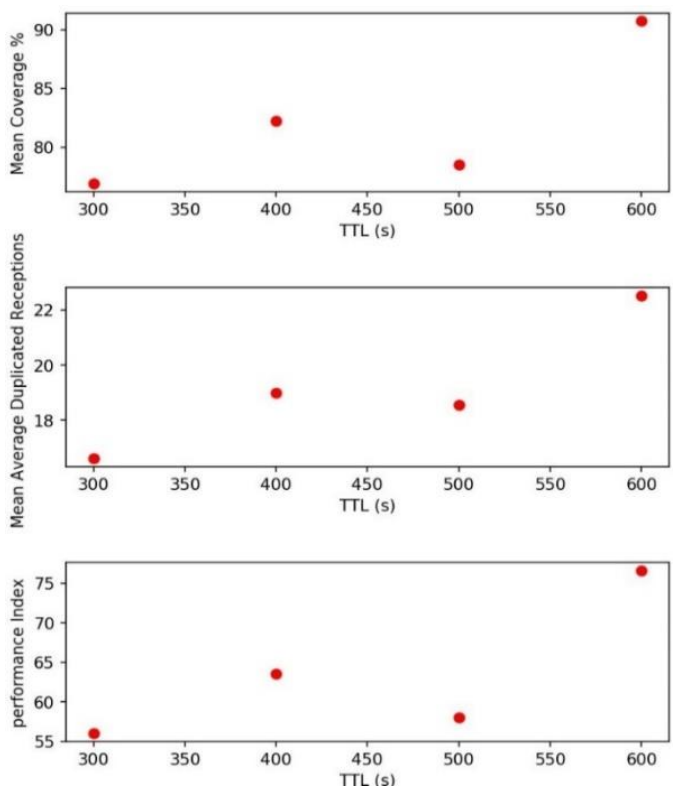

Fig. 8. Opportunistic DHVN protocol simulation results for different TTL duration in rural environment.

\section{REFERENCES}

[1] L. Aparecido, "Data dissemination in vehicular networks: Challenges, solutions, and future perspectives", in 2015 7th International Conference on New Technologies, Mobility and Security (NTMS), 2015, p. 1-5.

[2] U. Hayat, R. Iqbal, et J. Diab, "Eliminating Broadcast Storming in Vehicular Ad-Hoc Networks", Int. J. Adv. Comput. Sci. Appl., vol. 7, no $1,2016$.

[3] S. Mehar, S. M. Senouci, et G. Rémy, "Dissemination protocol for Heterogeneous Cooperative Vehicular Networks", in 2012 IFIP Wireless Days, 2012, p. 1-6.

[4] S. Panichpapiboon et W. Pattara-Atikom, "A Review of Information
Dissemination Protocols for Vehicular Ad Hoc Networks", Commun. Surv. Tutor. IEEE, vol. 14, no 3, p. 784-798, Quarter.

[5] I. Achour, T. Bejaoui, et S. Tabbane, "Network coding approach for vehicle-to-vehicle communication: Principles, protocols and benefits", in 2014 22nd International Conference on Software, Telecommunications and Computer Networks (SoftCOM), 2014, p. 154-159.

[6] J. A. Sanguesa, M. Fogue, P. Garrido, F. J. Martinez, J.-C. Cano, et C. T. Calafate, "A Survey and Comparative Study of Broadcast Warning Message Dissemination Schemes for VANETs", Mobile Information Systems, 2016. [En ligne]. Disponible sur: https://www.hindawi.com/journals/misy/2016/8714142/. [Consulté le: 27déc-2017].

[7] A. Mchergui, T. Moulahi, B. Alaya, et S. Nasri, "A Survey and Comparative Study of QoS Aware Broadcasting Techniques in VANET", Telecommun Syst, vol. 66, no 2, p. 253-281, oct. 2017.

[8] R. Ghebleh, "A comparative classification of information dissemination approaches in vehicular ad hoc networks from distinctive viewpoints: A survey”, Comput. Netw., vol. 131, p. 15-37, févr. 2018.

[9] M.-T. Sun, W.-C. Feng, T.-H. Lai, K. Yamada, H. Okada, et K. Fujimura, "GPS-based message broadcast for adaptive inter-vehicle communications", in Vehicular Technology Conference, 2000. IEEEVTS Fall VTC 2000. 52nd, 2000, vol. 6, p. 2685-2692.

[10] R. C. Voicu, H. I. Abbasi, H. Fang, B. Kihei, J. A. Copeland, et Y. Chang, "Fast and reliable broadcasting in VANETs using SNR with ACK decoupling", in 2014 IEEE International Conference on Communications (ICC), 2014, p. 574-579.

[11] N. Ababneh et H. Labiod, "Safety message dissemination in VANETs: Flooding or trajectory-based?", in Ad Hoc Networking Workshop (MedHoc-Net), 2010 The 9th IFIP Annual Mediterranean, 2010, p. 1-8.

[12] S. Zemouri, S. Djahel, et J. Murphy, "Short paper: Road-Casting: A new distributed dissemination protocol for safety messages in urban areas", in 2013 IEEE Vehicular Networking Conference (VNC), 2013, p. 234-237.

[13] M. O. Cherif, S.-M. Secouci, et B. Ducourthial, "How to disseminate vehicular data efficiently in both highway and urban environments?", in 2010 IEEE 6th International Conference on Wireless and Mobile Computing, Networking and Communications (WiMob), 2010, p. 165171.

[14] B. Tian, K. M. Hou, et J. Li, "TrAD: Traffic Adaptive Data Dissemination Protocol for Both Urban and Highway VANETs", in 2016 IEEE 30th International Conference on Advanced Information Networking and Applications (AINA), 2016, p. 724-731.

[15] M. Rathod, I. Mahgoub, et M. Slavik, "A hybrid data dissemination scheme for VANETs", in Wireless Days (WD), 2011 IFIP, 2011, p. 1-7.

[16] J. Balen, J. Matijaš, et G. Martinović, "Simulation and testing of VANET protocols", Tridesettreći Skup O Promet. Sustavima Med Junarodnim Sudjelov. Autom. U Prometu KoREMA, p. 5-8, 2013.

[17] C. Sommer, R. German, et F. Dressler, "Bidirectionally Coupled Network and Road Traffic Simulation for Improved IVC Analysis", IEEE Trans. Mob. Comput., vol. 10, no 1, p. 3-15, janv. 2011.

[18] C. Sommer, Z. Yao, R. German, et F. Dressler, "Simulating the influence of IVC on road traffic using bidirectionally coupled simulators", in INFOCOM Workshops 2008, IEEE, 2008, p. 1-6.

[19] V. Kumar et al., "itetris: Adaptation of its technologies for large scale integrated simulation", in Vehicular Technology Conference (VTC 2010Spring), 2010 IEEE 71st, 2010, p. 1-5.

[20] J. Harri, F. Filali, et C. Bonnet, "A framework for mobility models generation and its application to inter-vehicular networks", in 2005 International Conference on Wireless Networks, Communications and Mobile Computing, 2005, vol. 1, p. 42-47 vol.1.

[21] J. Harri, F. Filali, et C. Bonnet, "Mobility models for vehicular ad hoc networks: a survey and taxonomy", IEEE Commun. Surv. Tutor., vol. 11, no 4, p. 19-41, 2009.

[22] R. Fernandes, F. Vieira, et M. Ferreira, "VNS: An integrated framework for vehicular networks simulation", in Vehicular Networking Conference (VNC), 2012 IEEE, 2012, p. 195-202 